\section{Propuesta turística para el circuito Marvilla basada en los registros de la avifauna del sitio Ramsar Los Pantanos de Villa}

Tourist proposal for the Marvilla Circuit based on the records of the birdlife for Pantanos de Villa Ramsar Site

Sofía Chung-Velásquez ${ }^{1,3 *}$ y Hayde Lopez-Manrique ${ }^{2,3} \odot$

1 Facultad de Ciencias Biológicas y Veterinarias, Universidad Científica del Sur. Lima, Perú.

2 Facultad de Ingeniería Geográfica, Ambiental y Ecoturismo, Universidad Nacional Federico Villarreal. Lima, Perú.

3 Área Natural Protegida de los Pantanos de Villa, Prohvilla. Lima, Perú.

\section{SOUTH} SUSTAINABILITY

Citar como: Chung-Velásquez, S. y López-Manrique, H. (2021). «Propuesta turística para el circuito Marvilla basada en los registros de la avifauna del sitio Ramsar Los Pantanos de Villa». South Sustainability, 2(2), e039. doi: https://doi.org/10.21142/SS-02022021-pb001

Artículo recibido: 18/5/2021 Revisado por pares

Artículo aceptado: 21/7/2021

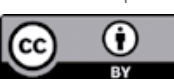

(C) Las autoras, 2021. Publicado por la Universidad Científica del Sur (Lima, Perú)

*E-mail de correspondencia: sofiacchv@gmail.com

\section{RESUMEN}

En el área ecológica metropolitana los Pantanos de Villa se encuentra el destacado circuito turístico Marvilla, cuyo trayecto cruza parte del litoral de Chorrillos. En su recorrido se puede observar gran variedad de especies de aves, tanto residentes como migratorias, que aprovechan esta área para alimentarse, reposar e incluso como lugar de nidificación. Dadas las características de este circuito, es importante el planteamiento de medidas adecuadas que permitan simultáneamente la protección de las aves y las visitas guiadas. Después de analizar los registros de las aves en los Pantanos de Villa durante 2020, se propone las visitas durante los meses de diciembre a marzo como ideales para las actividades turísticas. Se recomienda reducir el aforo de personas durante la época reproductiva y tomar en cuenta los estresores antropogénicos, que pueden llevar al cambio de estructura y abundancia de la avifauna. Asimismo, se resalta la labor de registros sistemáticos que permitieron el análisis de la información para tomar precauciones y plantear gestiones adecuadas.

Palabras clave: migración, estresores, invasión, humedal, monitoreo, análisis

\section{ABSTRACT}

In the metropolitan ecological area Los Pantanos de Villa is located the «Marvilla» tourist circuit, an outstanding tourist circuit whose route crosses part of the Chorrillos coastline. In the tour of the circuit you can see a variety of bird species, both resident and migratory, that take advantage of this area to feed, rest, and even as a nesting place. Given the characteristics of this circuit, it is important to plan appropriate measures that simultaneously allow the protection of birds and guided visits. After analyzing the records of the birds in the Pantanos de Villa during 2020, visits during the months of december-march are proposed as ideal for tourist activities. It is recommended to reduce the capacity of people during the reproductive season, it is requested to take into account the anthropogenic stressors that can lead to the change of structure and abundance of the birdlife, and the work of systematic records that allowed the analysis of the information to be taken is highlighted. precautions and take appropriate steps.

Keywords: migration, stressors, invasion, wetland, monitoring, analysis 


\section{Introducción}

La actividad turística tomó particular relevancia en los últimos años, especialmente desde un punto de vista medioambiental (Bötsch, 2018). El fenómeno turístico es un tema particularmente complejo (Cianga, 2017), que está fuertemente relacionado con los viajes y con actividades humanas que generaron un gran impacto en distintas áreas humanas, entre las que destaca el ámbito socioeconómico (Bunguez, 2016).

Los responsables de la implementación de políticas y decisiones deben ser conscientes de las interconexiones que existen entre una actividad u otra, y del valor que tienen estas para satisfacer y preservar las futuras necesidades sociales, económicas y ambientales en el correcto mantenimiento de ecosistemas (Erkol, 2017).

Entre las ramas del turismo destaca el ecoturismo, también conocido como turismo sustentable, que es relevante por tener un enfoque ambiental responsable que vela por la preservación y conservación del área silvestre visitada, los usos adecuados de los recursos que se encuentran en el lugar y de los que se dispone, y la concientización del área delimitada y de los impactos que tendría su alteración en la naturaleza o cultura de la comunidad visitada (Rebollo, 2012).

Un lugar ecoturístico destacado son los Pantanos de Villa, un área natural protegida que cuenta con reconocimientos de categoría nacional e internacional, ubicada en Lima (Perú). Presenta abundantes especies de flora y fauna, y por ello se busca promover en el sitio la conservación y el uso racional (Ramsar, 2015). Esto lo hace uno de los principales atractivos turísticos cerca de la ciudad. Funciona con fines recreativos y científicos (Carhuas, 2020).

Entre las rutas que ofrece Pantanos de Villa encontramos el circuito turístico Marvilla, que cuenta con una de las lagunas más grandes del humedal y cuyo trayecto cruza parte del litoral de Chorrillos. En el recorrido del circuito se puede encontrar variedad de especies de aves, tanto residentes como migratorias, que reposan a los alrededores de su laguna. El circuito Marvilla recibe por año cientos de turistas y estudiantes, que representan parte de los ingresos económicos al área (Amaro et al., 2017). Las aves tienen acceso directo a la zona litoral, lo cual les permite realizar actividades de alimentación y reposo. También por esta razón utilizan el recorrido y sus alrededores de forma no sistemática para las actividades de reproducción y anidamiento (Arenas et al., 2020).

Por ello, es importante el planteamiento de medidas adecuadas que permitan simultáneamente la protección de las aves y sus actividades, y las visitas guiadas realizadas en la zona.

El objetivo es analizar los datos existentes de las aves en este circuito de los Pantanos de Villa, a fin de determinar las fechas más propicias para la realización de las actividades turísticas, teniendo como principal enfoque la protección y conservación de las aves.

\section{Enfoque y discusión}

En los Pantanos de Villa existen alrededor de 211 especies de aves, 97 residentes y 114 migratorias (Pulido, 2020). Distintas aves migratorias llegan a los Pantanos de Villa, específicamente al circuito Marvilla, donde descansan y se alimentan, como parte de su corredor migratorio. A su vez, las aves residentes obtienen refugio y alimentos de forma permanente en esta zona. La mayoría proceden de distintos lugares del hemisferio norte, como el Ártico canadiense y Alaska, y del hemisferio sur (Torres et al., 2006). Debido a la gran cantidad de aves migratorias, se ha reconocido su importancia ecológica internacional (Pulido et al., 2018b).

Gracias a la importante labor de las instituciones como Prohvilla, de voluntarios, y autoridades competentes como Sernanp, existen registros de las aves encontradas en el circuito Marvilla durante el periodo 2016-2020 (Pulido, 2018). Se logró establecer la presencia de hasta 18 especies de aves simultáneamente entre migratorias y residentes durante 2020 (tabla 1).

Entre las 18 especies registradas diferenciamos a las aves migratorias. Se tienen registros de zarapito trinador (Numenius phaeopus), playero blanco (Calidris alba), rayador (Rynchops niger), gaviota de Franklin (Leucophaeus pipixcan), gaviota peruana (Larus belcheri), gaviota dominicana (Larus dominicanus), piquero común (Sula variegata), guanay (Phalacrocorax bougainvillii) y pelícano peruano (Pelecanus thagus). Es fundamental comprender que la presencia de las aves migratorias está influenciada por su estacionalidad biológica. Así, pueden encontrarse determinadas aves, en el humedal, en ciertas fechas del año en el camino de su migración (Arya, 2019).

Tal es el caso de una las aves migratorias más conocida de los Pantanos deVilla, la gaviota de Franklin (Leucophaeus pipixcan), que se aglomera frecuentemente en el circuito Marvilla, hasta ser una de sus aves estacionales más abundantes y representativas, con 23956 aves registradas en 2020. Esta especie arriba a los Pantanos de Villa durante los meses de noviembre y diciembre, para quedarse durante la temporada de verano hasta finales de marzo o inicios de abril. Cubre casi en su totalidad la zona litoral del circuito Marvilla. De igual modo, se presenta al ave migratoria boreal (Mb) rayador (Rynchops niger), que anida en el hemisferio norte (BirdLife, 2016) y es más frecuentemente vista entre septiembre y abril. También se puede observar al ave migrante boreal (Mb) zarapito trinador (Numenius phaeopus), encontrada en el Perú igualmente entre septiembre y abril (Schulenberg, 2007).

Por otro lado, en este circuito también existe un número considerable de aves residentes que nidifican, 
permanecen en el sitio y solo realizan desplazamientos locales durante todo el año (Martínez y Petracci, 2017). Estas especies son muy importantes para los procesos ecológicos del área en donde se encuentren, ya que suelen ser dispersoras de semillas (como es el caso de los integrantes de la familia Anatidae), de forma que se logra la recuperación de áreas naturales que con el paso del tiempo pudieron verse afectadas. Además, funcionan como controladores biológicos naturales, como es el caso de los integrantes de la familia Rallidae y de otras especies como insectos (Osbahr y Constanza, 2014; Pulido et al., 2018a).

Tabla 1. Grupo taxonómico (familia), código asignado, nombre científico y común, migratorio/residente $(\mathrm{R}=$ residente, $\mathrm{Mb}=$ migrante boreal, $\mathrm{Ml}=$ migrante local) del ave y número de individuos localizados en los transectos T5 y T6 a los alrededores del circuito Marvilla en 2020.

\begin{tabular}{|c|c|c|c|c|}
\hline Familia & $\begin{array}{c}\text { Nombre } \\
\text { científico }\end{array}$ & $\begin{array}{l}\text { Nombre } \\
\text { común }\end{array}$ & $\begin{array}{l}\text { Residente/ } \\
\text { migratorio }\end{array}$ & $\begin{array}{l}\text { Total } \\
2020\end{array}$ \\
\hline Anatidae & $\begin{array}{c}\text { Spatula } \\
\text { cyanoptera }\end{array}$ & $\begin{array}{c}\text { Pato } \\
\text { colorado }\end{array}$ & $\mathrm{R}$ & 748 \\
\hline Anatidae & $\begin{array}{c}\text { Anas } \\
\text { bahamensis }\end{array}$ & $\begin{array}{c}\text { Pato } \\
\text { gargantillo }\end{array}$ & $\mathrm{R}$ & 294 \\
\hline Rallidae & $\begin{array}{c}\text { Gallinula } \\
\text { galeata }\end{array}$ & $\begin{array}{l}\text { Polla de } \\
\text { agua }\end{array}$ & $\mathrm{R}$ & 554 \\
\hline Haematopodidae & $\begin{array}{c}\text { Haematopus } \\
\text { palliatus }\end{array}$ & $\begin{array}{l}\text { Ostrero } \\
\text { común }\end{array}$ & $\mathrm{R}$ & 535 \\
\hline Scolopacidae & $\begin{array}{l}\text { Numenius } \\
\text { phaeopus }\end{array}$ & $\begin{array}{l}\text { Zarapito } \\
\text { trinador }\end{array}$ & $\mathrm{Mb}$ & 357 \\
\hline Scolopacidae & Calidris alba & $\begin{array}{l}\text { Playero } \\
\text { blanco }\end{array}$ & $\mathrm{Mb}$ & 10 \\
\hline Rhynchopidae & Rynchops niger & Rayador & $\mathrm{Mb}$ & 2558 \\
\hline Laridae & $\begin{array}{c}\text { Chroicocephalus } \\
\text { cirrocephalus }\end{array}$ & $\begin{array}{c}\text { Gaviota } \\
\text { capucho } \\
\text { gris }\end{array}$ & $\mathrm{R}$ & 358 \\
\hline Laridae & $\begin{array}{c}\text { Leucophaeus } \\
\text { pipixcan }\end{array}$ & $\begin{array}{l}\text { Gaviota de } \\
\text { Franklin }\end{array}$ & $\mathrm{Mb}$ & $\begin{array}{c}23 \\
956\end{array}$ \\
\hline Laridae & Larus belcheri & $\begin{array}{l}\text { Gaviota } \\
\text { peruana }\end{array}$ & MI & 794 \\
\hline Laridae & $\begin{array}{c}\text { Larus } \\
\text { dominicanus }\end{array}$ & $\begin{array}{c}\text { Gaviota } \\
\text { dominicana }\end{array}$ & MI & 617 \\
\hline Sulidae & Sula variegata & $\begin{array}{l}\text { Piquero } \\
\text { común }\end{array}$ & MI & 2087 \\
\hline Phalacrocoracidae & $\begin{array}{c}\text { Phalacrocorax } \\
\text { brasilianus }\end{array}$ & $\begin{array}{c}\text { Cushuri, } \\
\text { cormorán, } \\
\text { cuervo de } \\
\text { mar }\end{array}$ & $\mathrm{R}$ & 1225 \\
\hline Phalacrocoracidae & $\begin{array}{c}\text { Phalacrocorax } \\
\text { bougainvillii }\end{array}$ & Guanay & MI & 9417 \\
\hline Pelecanidae & $\begin{array}{c}\text { Pelecanus } \\
\text { thagus }\end{array}$ & $\begin{array}{l}\text { Pelícano } \\
\text { peruano }\end{array}$ & $\mathrm{MI}$ & 185 \\
\hline Threskionithidae & $\begin{array}{l}\text { Plegadis } \\
\text { ridgwayi }\end{array}$ & Yanavico & $\mathrm{R}$ & 139 \\
\hline \multicolumn{4}{|c|}{ Total } & $\begin{array}{c}43 \\
834\end{array}$ \\
\hline
\end{tabular}

En el circuito Marvilla, según datos recolectados en 2020 (tabla 1), se observaron siete especies frecuentes de aves residentes: el pato colorado (Spatula cyanoptera), el pato gargantillo (Anas bahamensis), la polla de agua (Gallinula galeata), el ostrero común (Haematopus palliatus), la gaviota capucho gris (Chroicocephalus cirrocephalus), el cormorán (Phalacrocorax brasilianus) y el yanavico (Plegadis ridgwayi). La zona litoral del circuito Marvilla es de principal importancia para estas aves, que no solo usan esta zona para desplazarse o alimentarse, sino también como ubicación para la colocación de sus nidos (Figueroa, 2016). Ambas zonas, la ruta del circuito Marvilla y su zona litoral, están expuestas al paso frecuente de personas por el circuito por los turistas, y la zona litoral, por el paso libre de transeúntes. Por lo tanto, el éxito de anidación y eclosión de huevos de las aves residentes, particularmente del ostrero común, se ve limitado por la actividad antrópica (Arenas, 2020).

Según los datos obtenidos, se aprecia que entre las especies residentes más representativas del circuito Marvilla está el ostrero común (Haematopus palliatus) (Arenas et al., 2020), que utiliza zonas del circuito turístico

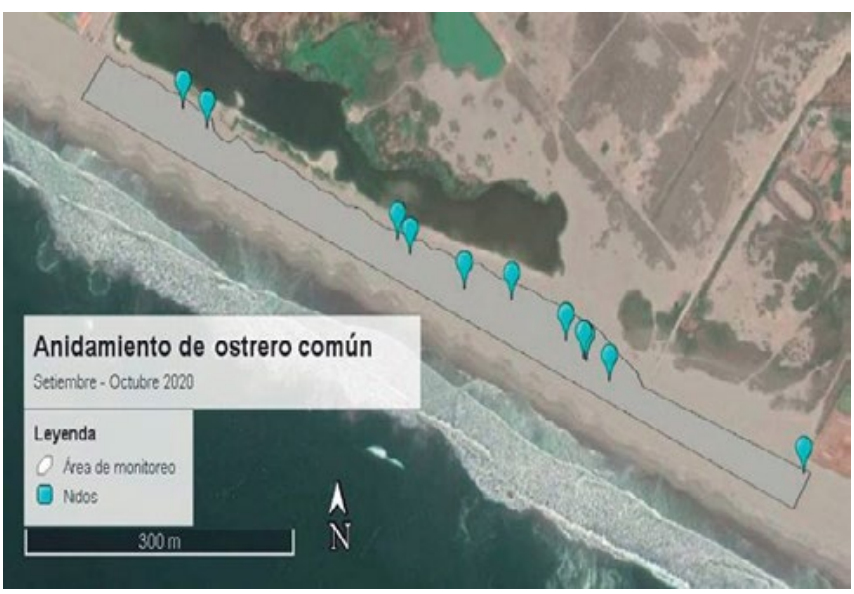

Figura 1. Mapa de ubicación de los nidos del ostrero común encontrados de septiembre a octubre de 2020 en el circuito Marvilla (Arenas, 2020).

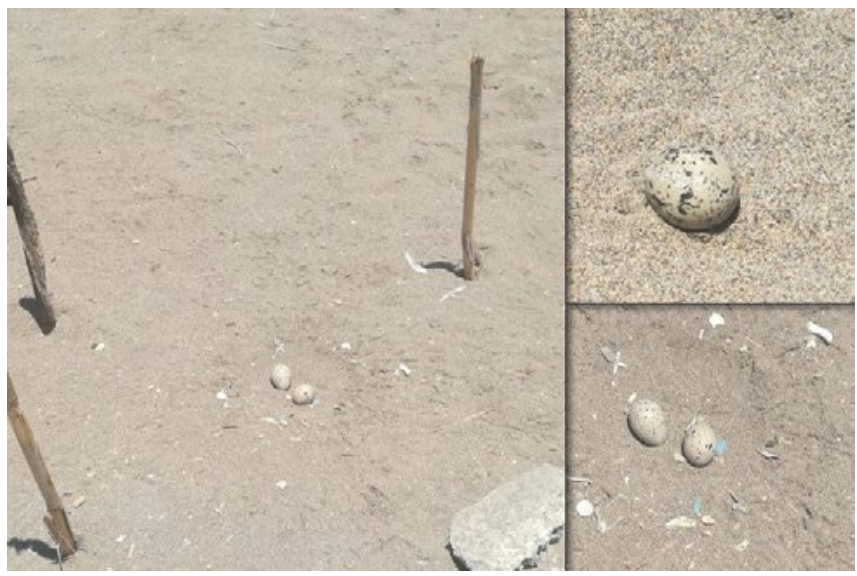

Figura 2. Anidamiento del ostrero común en la zona litoral peruana. Fuente: Elaboración propia. 
y parte de la zona litoral peruana para la colocación de sus nidos (figura 1), de forma no sistematizada (Figueroa, 2016).

Para esta especie se reportaron puestas durante todo el año después de un estudio a lo largo del litoral peruano (Figueroa, 2016), que coincide con el encuentro de huevos en el circuito Marvilla de Pantanos de Villa (figura 2). Por otra parte, está el pato colorado (Spatula cyanoptera), que suele reproducirse durante los meses de abril y julio. También resalta la polla de agua común (Gallinula galeata), que es abundante en el área y suele reproducirse de octubre a enero.

Cabe resaltar que la preservación de un humedal incluye distintos aspectos que se deben tener en cuenta, incluyendo la conservación de especies, el uso responsable, racional y gestionado de sus recursos, y la educación de la población adyacente y los visitantes, a fin de no alterar la fauna y flora de manera negativa (Kumar, 2014).

En el circuito Marvilla se conocen diversos estresores ambientales que enfrentan las aves, especialmente las residentes. La zona adyacente a la laguna, la zona litoral que las aves aprovechan como zona de nidificación, es la más expuesta a estos estresores, por verse pobremente delimitada. Entre estos tenemos el uso irresponsable del área con presencia intrusiva de turistas, fotógrafos, pobladores, excursionistas, cuatrimotos y caballos, que tienen poco o nulo conocimiento de la presencia y actividades de las aves y sus nidos, y de la función ecológica de Pantanos de Villa como lugar turístico, lo que genera impacto negativo antropogénico.

Se creía que los únicos estresores de aves se debían a la alteración o disturbio de sus ambientes (Remacha et al., 2016). Sin embargo, se ha demostrado que la presencia humana, el ruido generado y las actividades realizadas adyacentes a ellas pueden también efectuar aspectos conductuales de la avifauna (Steven, 2011; Lindsay et al., 2008). Sin embargo, diversos estudios (Curiel-Durán, 2017) demostraron que la actividad turística tiene importantes efectos directos e indirectos en las aves, entre los que destaca el impacto en su comportamiento y fisiología. Resulta de particular atención el elevado estrés ambiental y hormonal al que son expuestas, que pueden tener efectos graves como la alteración de su éxito reproductivo (Lezama, 2010).

\section{Conclusión y propuesta turística}

Las aves, como parte de la comunidad ecológica en Pantanos de Villa, son esenciales desde un punto de vista ecológico y turístico de la zona. La conservación del humedal es indispensable para la protección y conservación de las aves. La mantención de su hábitat, así como la disminución de estresores ambientales, a través de señalizaciones y delimitaciones correctas del área, se vuelven tareas vitales para asegurar su protección, tanto de las aves migratorias como residentes (NAWCC, 2020).

Gracias al análisis de los monitoreos por parte del personal de Prohvilla y de acuerdo con la información bibliográfica estudiada, planteamos las siguientes recomendaciones. Las visitas de diciembre a marzo son ideales para las actividades turísticas o afines, que buscan encontrar especies de aves migratorias y residentes. Asimismo, se recomienda reducir el aforo de personas durante la época reproductiva de anidamiento de las aves residentes, como es el caso del ostrero común, Haematopus palliatus. Según diversos estudios, esta especie suele anidar entre abril y julio, aunque en Lima también se registraron casos de anidamiento en enero y febrero (Figueroa, 2016). Para el caso particular del ostrero común, sería ideal el acompañamiento permanente de un guía especializado, que evite pasar por zonas de anidamiento ocasionales. Finalmente, una adecuada señalización del circuito y la delimitación correcta de sus espacios permitirán a los turistas el adecuado uso del circuito Marvilla, a la vez que se afectará lo mínimo posible a la fauna y la flora.

De igual modo, desde un punto de vista ecológico, se deben tomar en cuenta a los estresores antropogénicos, que pueden llevar al cambio de estructura y abundancia de la avifauna a largo o mediano plazo (Sousa, 2009). Para favorecer la conservación del circuito Marvilla y las aves, es necesario velar por mantener las condiciones ecológicas adecuadas, ya que los cambios podrían llevar a problemas ecológicos más grandes (Stewart, 2016). Todo ello son consideraciones para la creación de una gestión responsable.

Los estudios realizados nos llevan a pensar que uno de los mejores usos del circuito es el de fines investigativos, por ser de particular interés para el estudio biológico de aves residentes como migratorias.

Se resalta la labor de registros sistemáticos que permitieron y permiten un adecuado análisis de la información para tomar precauciones y plantear gestiones adecuadas en el desarrollo de actividades turísticas de manera propicia dirigidas a los turistas y la conservación de aves. Los registros funcionan como indicadores ecológicos de las condiciones de los Pantanos de Villa a largo plazo y ayudan a detectar y evaluar cambios en las condiciones ambientales, así como para determinar los mejores métodos para su recopilación (Canterbury, 2000). Se anima a continuar con la actividad de monitoreo sistematizada y enfocada para proveer el valioso recurso que es la información para el apoyo de largo plazo de gestión programas, informes y estudios sobre ecosistemas (Lovett et al., 2007) como los Pantanos de Villa.

\section{Contribución de autoría}

Ambas autoras contribuyeron de igual manera en la recopilación y el análisis de información, y la elaboración y la redacción del manuscrito. 


\section{Fuente de financiamiento}

Programa de Pasantías de Prohvilla 2021-1.

\section{Potenciales conflictos de interés}

No existe ningún potencial conflicto de interés en este artículo.

\section{Referencias bibliográficas}

Amaro, L. y Goyoneche, G. (2017). «Nesting at the Pantanos de Villa wildlife refuge 2007-2009, Lima-Perú». The Biologist (Lima), 15(1), pp. 155-171.

Arenas, A., Camarena, N., Ponce, J. y Corillo, A. (2020). «Éxito reproductivo del Haematopus palliatus, ostrero común, y actividades antrópicas en el Circuito Marvilla, Pantanos de Villa, Lima, Perú». South Sustainability, 1(2), e020. doi: 10.21142/SS-0102-2020-020

Arya, A. K., Bhatt, D., Singh, A., Saini, V., Verma, P., Rathi, R. y Bhatnagar, P. (2019). «Diversity and status of migratory and resident wetland birds in Haridwar, Uttarakhand, India». Journal of Applied and Natural Science, 11(3), pp. 732-737. Disponible en: https:// doi.org/10.31018jans.v11i3.2085

BirdLife International (2016). Rynchops niger. The IUCN Red List of Threatened Species 2016: e.T22694256A93442996. Disponible en: https://dx.doi.org/10.2305/IUCN.UK.2016-3.RLTS. T22694256A93442996.en

Bötsch, Y., Tablado, Z., Scherl, D., Kéry, M, Graf, R. y Jenni, L. (2018). «Effect of recreational trails on forest birds: Human presence matters». Frontiers in Ecology and Evolution, 12. Disponible en: https://www.frontiersin.org/articles/10.3389/fevo.2018.00175/full

Bunghez, C. L. (2016). «The importance of tourism to a destination's economy». Journal of Eastern Europe Research in Business \& Economics, 2016 (2016). doi: 10.5171/2016.143495. Disponible en: https://ibimapublishing.com/articles/JEERBE/2016/143495/143495. pdf

Canterbury, G., Martin, T., Petit, D., Petit, L. Y Bradfords, D. (2000). «Bird communities and habitat as ecological indicators of forest condition in regional monitoring». Conservation Biology, 14(2). Disponible en: http://www.umt.edu/mcwru/documents/Martin Publications/Reprint574.pdf

Carhuas Conde, J. y Jacinto Castro M. E. (2020). Análisis de la situación del turismo de observación de aves en el Refugio Vida Silvestre los Pantanos de Villa, Lima (2019). Trabajo de investigación de bachiller en Turismo Sostenible y Hotelería. Universidad Científica del Sur. Disponible en: https://hdl.handle.net/20.500.12805/1245

Cianga, N. (2017). "The impact of tourism activities. A point of view». Risks and Catastrophes Journal, 20, pp. 25-40. doi: 10.24193/ RCJ2017 02. Disponible en: https://www.researchgate.net/ publication/318881678_The_impact_of_tourism_activities_A_point_ of_view

Curiel-Durán, H., Lara, C., Castillo Guevara, C. y Ortiz-Pulido, R. (2017). «El impacto en las aves por el turismo de naturaleza: una mini revisión». Mexican Journal of Biotechnology, 2(1), pp. 37-45.
Erkol Bayram, G., Karaçar, E. y Bayram, A. T. (2017). «The importance of ecotourism consciousness on tour guiding education». Karabük Üniversitesi, Sosyal Bilimler Enstitüsü Dergisi, 3, pp. 40-50. Disponible en: https://www.researchgate.net/publication/322314891 The Importance of Ecotourism Consciousness on Tour Guiding Education

Figueroa, J. y Stucchi, M. (2016). «Biología reproductiva del ostrero americano (Haematopus palliatus pitanay) en el Perú». Revista Chilena de Ornitología, 22(2), pp. 171-183.

Kumar, A. y Kanaujia, A. (2014). «Wetlands: Significance, threats and their conservation». Disponible en: https://www.researchgate.net/ publication/327816889_Wetlands_Significance_Threats_and their Conservation

Lindsay, K., Craig, J. y Low, M. (2008). «Tourism and conservation: the effects of track proximity on avian reproductive success and nest selection in an open sanctuary». Tourism Management, 29, 730e739.

Lovett, G. M., Burns, D. A., Driscoll, C. T., Jenkins, J. C., Mitchell, M. J., Rustad, L., Shanley, J. B., Likens, G. E. y Haeuber, R. (2007). «Who needs environmental monitoring? Review». Frontiers in Ecology and the Environment, 5(5), pp- 253-260.

Marsano Delgado, J. M. (2017). «El impacto económico del turismo en el Perú 1990-2015». Turismo y Patrimonio, (10), pp. 155-168. https:// doi.org/10.24265/turpatrim.2016.n10.12

Martínez, N. (2017). Ecología de aves playeras migratorias durante la invernada, migración, sobre-veraneo en Bahía Samborombón, Buenos Aires, Argentina. Tesis doctoral en Ciencias Biológicas. Universidad de Buenos Aires. Disponible en: http://digital.bl.fcen.uba.ar/ Download/Tesis/Tesis_6043_MartinezCurci.pdf

NAWCC. (2020). «Protecting, restoring and enhancing habitat for migratory birds». Disponible en: www.fws.gov/birds/grants/northamerican-wetland-conservation-act.php

Osbahr, K. y Constanza, N. (2014). «Uso de hábitat de la avifauna en el humedal Guaymaral (Cundinamarca, Colombia)». Revista U. D. C. A. Actualidad \& Divulgación Científica, 9(2), pp. 157-168. Disponible en: https://repository.udca.edu.co/bitstream/handle/11158/2532/ Art\%C3\%ADculo.pdf?sequence=1\&isAllowed =y

Pulido, V. (2018). «One hundred and fifty years of keep records from Pantanos de Villa birds'». Revista Peruana de Biología, 25(3), 291-306. Disponible en: https://dx.doi.org/10.15381/rpb.v25i3.15212

Pulido, V., Salinas, L., Del Pino, J. y Arana, C. (2020). «Preferencia de hábitats y estacionalidad de las especies de aves de los Pantanos de Villa en Lima, Perú». Revista Peruana de Biología, 27(3), pp. 349-360. 
Pulido Capurro, V. y Bermúdez Díaz, L. (2018a). «Current conservation status of the habitats of the Pantanos de Villa, Lima, Perú». Arnaldoa, 25(2), pp. 679-702. Disponible en: https://dx.doi.org/ http://doi.org/10.22497/arnaldoa.252.25219

Pulido Capurro, V. y Bermúdez Díaz, L. (2018b). «Patrones de estacionalidad de las especies de aves residentes y migratorias de los Pantanos de Villa, Lima, Perú». Arnaldoa, 25(3). Disponible en: http://www.scielo.org.pe/scielo.php?script=sci_ arttext\&pid $=$ S2413-32992018000300018

Ramsar (2015). «La economía de los ecosistemas y la biodiversidad relativa al agua y los humedales. Resumen ejecutivo». TEEB, Aguas y Humedales. Disponible en: https://www.ramsar.org/sites/default/ files/documents/library/teeb_waterwetlands_execsum_2013-sp.pdf

Rebollo Soto, N. (2012). Ecoturismo. Tlalnepantla: Red Tercer Milenio. Disponible en: http://www.aliat.org.mx/BibliotecasDigitales/ economico administrativo/Ecoturismo.pdf

Remacha, C., Pérez-Tris, J. y Delgado, J. A. (2011). «Reducing visitors group size increases the number of birds during educational». Journal of Environmental Management, 92, pp. 1564-1568.

Schulenberg, T. S., Stotz, D. F., Lane, D. F., O'Neill, J. P. y Parker III, T. A. (2007). Aves del Perú. Birds of Peru. Lima: Museo de Historia Natural, Universidad Nacional Mayor de San Marcos.
Sousa, A., García-Barrón, L., Vetter, M., Agular, M., García-Murillo, P. y Morales, J. (2009). «El efecto acumulado de los impactos climáticos y antropogénicos sobre humedales del suroeste de España». Departamento de Biología y Ecología, Universidad de Sevilla. Disponible en: http://aeclim.org/wp-content/ uploads/2016/02/0095_PU-SA-VIII-2012-A_SOUSA.pdf

Stewart, R. E. (2016). «Technical aspects of wetlands as bird habitat». United States Geological Survey Water Supply Paper 2425. Disponible en: https://water.usgs.gov/nwsum/WSP2425/birdhabitat.html

Steven, R., Pickering, C. y Castley, J. G. (2011). «A review of the impacts of nature based recreation on birds». Journal of Environmental Management, 92, pp. 2287-2294.

Torres, M., Quinteros, Z. y Takano, F. (2006). «Variación temporal de la abundancia y diversidad de aves limícolas en el refugio de vida silvestre Pantanos de Villa, Perú». Ecología Aplicada, 5(1-2), 119-125. Recuperado en 29 de mayo de 2021, de http://www.scielo.org.pe/ scielo.php?script=sci_arttext\&pid=S1726-22162006000100016\&lng =es\&tlng=es

Ugalde Lezama, S., Alcantara, J. L., Valdez-Hernández, J., Ramirez, G., Velázquez-Mendoza, J. y Tarango-Arambula, L. (2010). «Riqueza, abundancia y diversidad de aves en un bosque templado con diferentes condiciones de perturbación». Agrociencia, 44(2), pp. 159-169. Disponible en: https://www.researchgate.net/ publication/262548967_Riqueza_abundancia_y_diversidad_de aves_en_un_bosque_templado_con_diferentes_condiciones_de perturbacion 